\title{
Preparation of Copper Nanofluids Using an Appropriate Technique
}

\author{
HAMID REZA GHORBANI* \\ Department of Chemical Engineering, Qaemshahr Branch, Islamic Azad University, Qaemshahr, Iran. \\ ${ }^{*}$ Corresponding author E-mail: hamidghorbani6@ gmail.com \\ http://dx.doi.org/10.13005/ojc/300467
}

(Received: September 25, 2014; Accepted: November 20, 2014)

\begin{abstract}
ABSRTACT
This work focuses on the synthesis of copper nanoparticles. The synthesis involves the use of copper nitrate, polyvinylpyrrolidone (pvp), dextrose and water as the copper precursor, stabilizing agent, reducing agent and solvent respectively. The nanoparticles were characterized by UV-visible spectroscopy, transmission electron microscopy (TEM) and dynamic light scattering (DLS). An absorption peak at $580 \mathrm{~nm}$ in Uv-Vis spectrophotometer was detected indicating the presence of copper nanoparticles. The DLS analysis showed Copper nanoparticles with size of 5$25 \mathrm{~nm}$.
\end{abstract}

Key words: Synthesis, Copper, Nanofluids, pvp.

\section{INTRODUCTION}

In the recent decades, metallic nanoparticles have been widely used in various industries due to their wide range of applications ${ }^{1,}$ ${ }^{2}$. Nanoparticles have unique electrical, physical, chemical and optical properties ${ }^{3}$. Nowadays copper is highly regarded due to its specific catalytic, optical, electrical and mechanical characteristics ${ }^{4}$. Copper nanoparticles have great applications as heat transfer systems, antibacterial materials, super strong materials, sensors, and catalysts ${ }^{5-7}$. Copper nanoparticles acts as an anti-biotic, anti-microbial, and anti-fungal agent when added to plastics, coatings, and textiles ${ }^{8-10}$. The advantages of copper nanoparticles are cheap, high yields in mild reaction conditions and have short reaction times compared to traditional catalysts ${ }^{11}$. Stable suspensions of nanometer solid particles in suitable solvents, socalled nanofluids, have shown enhanced thermal conductivity when compared with the fluid base ${ }^{12}$. In fact, nanofluids are dilute liquid suspensions of nanoparticles with at least one critical dimension smaller than $\sim 100 \mathrm{~nm}$. These fluids can be employed to increase the heat transfer rate in various applications. In among nanofluids, Copper nanofluids are important due their advantages and applications.

The synthesis methods of metal nanoparticles are basically divided into chemical, physical and biological methods ${ }^{132-16}$. In 2008, copper nanoparticles with a particle size less than $10 \mathrm{~nm}$ were synthesized by the reduction of $\mathrm{Cu} 2+$ 
in solution ${ }^{17}$. Procek et al. synthesized copper nanocrystals with a diameter of $14 \mathrm{~nm}$ using $\mathrm{Cu}$ reduction by sodium borohydride ${ }^{18}$. In another study, Chatterjee et al. used Cucl2 in the presence of gelatin as stabilizers to synthesize stable copper nanoparticles with a size of $50-60 \mathrm{~nm}^{19}$. In 2013, copper Nanocolloids with a size of $70-80 \mathrm{~nm}$ were synthesized using chemical reduction method $^{20}$.

This work presents the preparation of $\mathrm{Cu}$ nanofluids by simple route. In this study, we used copper nitrate as an initial reagent, dextrose as reducing agent, and polyvinyl pyrrolidone (pvp) as a capping agent.

\section{EXPERIMENTAL}

All the reagents used in the experiment were of analytical grade and were used without further purification. Copper nitrate, dextrose and polyvinyl pyrrolidone (PVP) were purchased from Sigma-Aldrich. Required amount of dextrose solution were added to a vessel containing copper nitrate solution and polyvinylpyrrolidone (pvp). Polyvinylpyrrolidone was added to stabilize nanoparticles and also to prevent their oxidation and accumulation.

The reaction between dextrose solution and $\mathrm{Cu}^{2+}$ ions were carried out in thermal conditions. After some minutes, the color of this solution changes to deep red. This color change indicates a possibility of copper nanoparticles formation. These nanoparticles were recovered by centrifugation and washed in ethanol twice. Copper nanoparticles produced were dispersed in ethylene glycol to prepare copper nanofluids. The prepared nanofluids were characterized by Uv-vis spectroscopy, transmission electron microscopy (TEM), and dynamic light scattering (DLS).

\section{RESULTS}

\section{UV-Vis spectral analysis}

Small metal nanoparticles exhibit the absorption of visible electromagnetic waves by the collective oscillation of conduction electrons at the surface ${ }^{21}$. This is known as the surface plasmon resonance effect. The interest in this effect is the possibility of using it as a tracer for the presence of metal nanoparticles with a simple UV-visible spectrometer.

The presence of copper nanoparticles in the suspension was checked by scanning its absorbance in the wavelength range of $500-750$

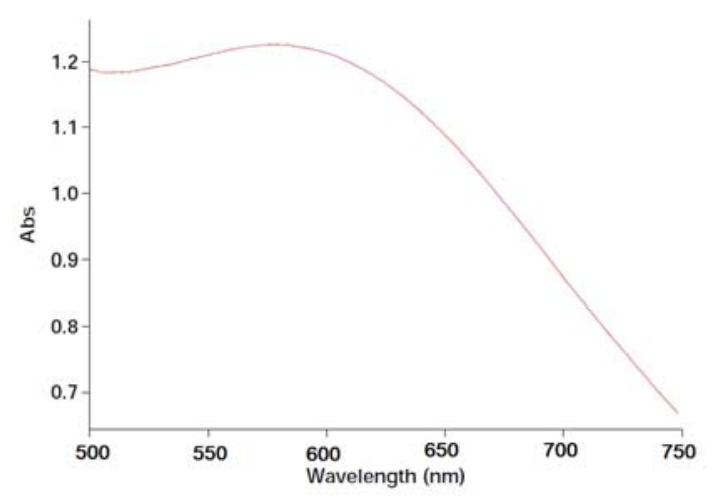

Fig.1: UV-vis spectra of copper nanofluids

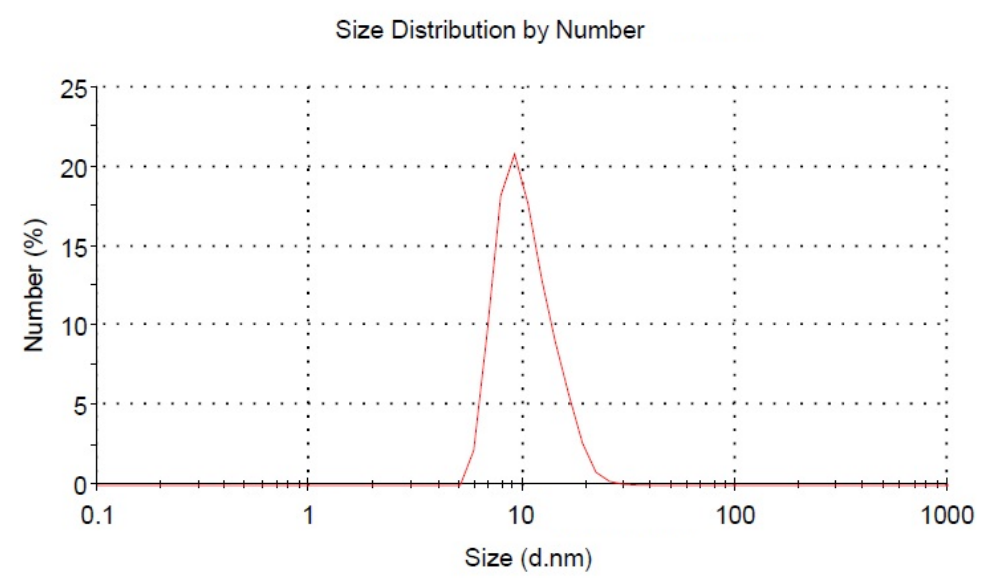

Fig. 2: The curve of size distribution by number (Copper nanofluids) 


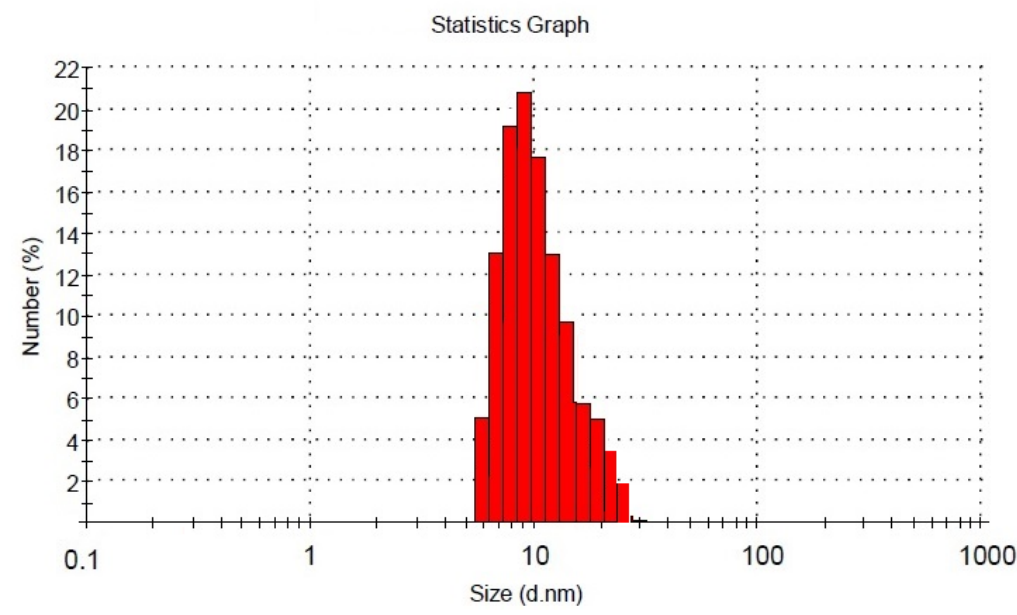

Fig. 3: The size distribution of copper nanoparticles by number

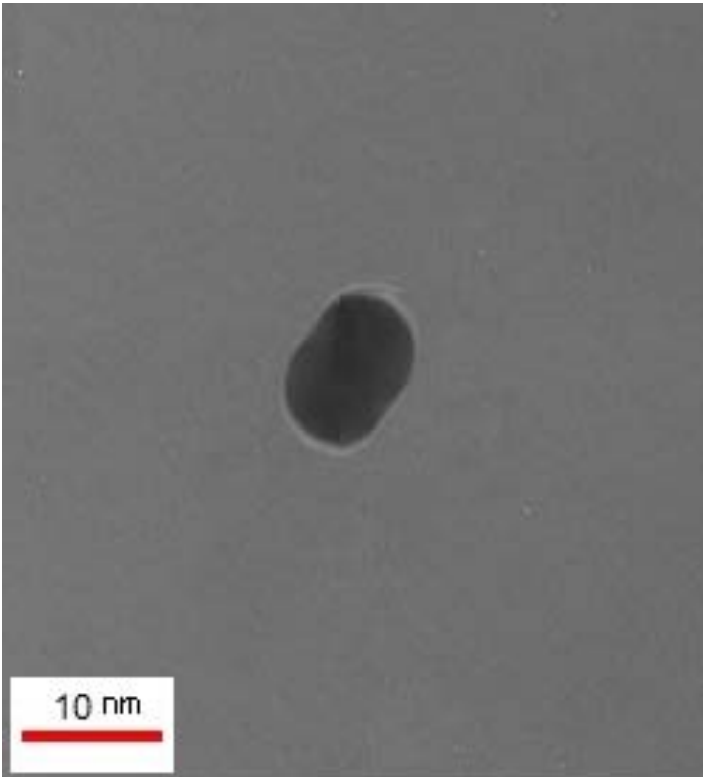

Fig. 4: Transmission electron micrographs (TEM) of copper nanoparticles

$\mathrm{nm}$ in a spectrophotometer. A UV/Vis spectrograph of the copper nanoparticle suspension can be seen in Fig. 1. A plasmon resonance peak at $\sim 580 \mathrm{~nm}$ is seen in the spectra. It has been suggested that this peak is associated with the formation of copper nanoparticles.

\section{DLS results}

Particle size is one of the most important characteristics of nanoparticles. The size distribution of synthesized nanoparticles and the average size was measured by using a dynamic light scattering (DLS) instrument. As is shown in figures 2 and 3 , average size of copper nanoparticles is about 10 $\mathrm{nm}$ and the distribution range of nanoparticles is about $20 \mathrm{~nm}$.

\section{TEM images}

Fig. 4 shows a representative TEM image of the produced copper nanoparticles. This image displays that the synthesized nanoparticles are non-agglomerated, and generally unlike in shape. Also images show and confirm copper nanoparticles production at nano-size.

\section{CONCLUSIONS}

In this work, copper nanoparticles were successfully synthesized by a reduction method using dextrose. Herein copper nitrate was reduced to copper and was simultaneously dispersed in the base fluid, in the presence of pvp. The synthesized nanoparticles were characterized by UV-Vis spectrophotometer, Transmission electron micrographs (TEM) and dynamic light scattering (DLS). The presence of metal nanoparticles (Cu0) is proved by the appearance of the surface plasmon resonance on nanofluids. We obtained relatively monodisperse copper nanoparticles with a size range of $5-25 \mathrm{~nm}$. 


\section{REFERENCES}

1. Parveen, S., Misra, R., Sahoo, SK., Nanomed Nanotechnol, 2012, 8(2), 147-166.

2. Yaghoubi, Z.; Motevalli, K. Int. J. Nano Dimens., 2015, 6(1), 45-54.

3. Mirza, A.Z.; Siddiqui, F.A. Int. Nano Lett., 2014, 4, 94.

4. Dhas, N.A.; Raj, C.P. ; Gedanken, A., Chem. Mater, 1998, 10, 1446-1452.

5. Gupta, R.; Sanotra, S.; Sheikh, H.N.; Kalsotra, B.L. J. Nanostructure Chem., 2013, 3, 41.

6. Samadi, S.; Khalilian, F.; Tabatabaee, A. J. Nanostructure Chem., 2014, 4, 84.

7. Ghane, M.; Sadeghi; B.; Jafari, A.R.; Paknejhad, A.R. Int. J. Nano Dimens., 2010, 1(1), 33-40.

8. El-Nahhal, M.; Zourab, S.M.; Kodeh, F.S.; Selmane, M.; Genois, I.; Babonneau, F. J. Int. Nano Lett., 2012, 2, 14.

9. Radhakrishnan, A.; Rejani, P.; Beena, B. Int. J. Nano Dimens., 2014, 5(6), 519-524.

10. Ghorbani, H.R. Chem Eng Commun, 2015, 10.1080/00986445.2014.950732.

11. Satlavati-Niasari, M.; Davar, F. Polyhedron, 2008, 27, 3514-3518.

12. Mishra, P. C.; Mukherjee, S.; Nayak, S.K.; Panda, A. Int. Nano Lett., 2014, DOI 10.1007/ s40089-014-0126-3.
13. Ghorbani, H. R.; Safekordi, A. A.; Attar, H.; Rezayat Sorkhabadi, S. M. Chem. Biochem. Eng. Q., 2011, 25 (3,) 317-326.

14. Sompech,S.; Nuntiya,A.; Orient.J.Chem, 2012, 28(1), 319-325.

15. Papaiah, S.; Seshadri Goud, T. E.; Devi Prasad, B. S.; Narasimha, G.; Vemana, K. Int. J. Nano Dimens., 2014, 5(2), 139-144.

16. Ghorbani, H.R. J. Nanostructure Chem., 2013, 3, 29.

17. Khodashenas, B.; Ghorbani, H.R. Int. J. Nano Dimens., 2015, 6(2), In press.

18. Ostaeva, G.Y.; Selishcheva, E.D.; Pautov, V.D.; Papisov, I.M. Polym Sci Ser B, 2008, 50(5-6), 147-149.

19. Prucek, R.; Kvítek, L.; Paná ek, A.; Van urová, L.; Soukupová, J.; Jan ík, D.; ZboYil, R. J Mat Chem, 2009, 19, 8463-8469.

20. Chatterjee, A.K.; Sarkar, R.K.; Chattopadhyay, A.P.; Aich, P.; Chakraborty, R.; Basu, T. Nanotechnology, 2012, 23(8), 85103-85113.

21. Kaminskiene, Z.; Prosy£evas, I.; Stonkute, J.; Guobiene, A. Acta Phys Pol A, 2013, 123, 111-114.

22. Link, S.; El-Sayed, M. A., Int. Rev. Phys. Chem., 2000, 19, 409-412. 